\title{
Pain and disability after first-time spinal fusion for lumbar degenerative disorders: a systematic review and meta-analysis
}

\author{
Niek Koenders ${ }^{1}$ (D) Alison Rushton ${ }^{2}$ (D) Martin L. Verra ${ }^{3}$ (D) Paul C. Willems ${ }^{4}$ (D) Thomas J. Hoogeboom $^{5}$ (D) \\ J. Bart Staal ${ }^{5,6}$ (D)
}

Received: 30 January 2018 / Accepted: 21 June 2018 / Published online: 11 July 2018

(c) The Author(s) 2018

\begin{abstract}
Purpose Lumbar spinal fusion (LSF) is frequently and increasingly used in lumbar degenerative disorders despite conflicting results and recommendations. A thorough understanding of patient outcomes after LSF is required to inform decisions regarding surgery and to improve post-surgery management. The current study aims to evaluate the course of pain and disability in patients with degenerative disorders of the lumbar spine after first-time LSF.

Methods A systematic review and meta-analysis of pain and disability outcomes in prospective cohort studies up to 31 March 2017 is identified in four electronic databases. Two independent researchers determined study eligibility, extracted data, and assessed risk of bias (modified Quality in Prognostics tool). A random effects model (maximum likelihood) was used to calculate means and $95 \%$ confidence intervals. The primary analysis was performed on complete data, and a sensitivity analysis was performed on all data.

Results Twenty-five studies ( $n=1777$ participants) were included. The mean (95\% confidence interval) Visual Analogue Scale (VAS) back pain ( $n=9$ studies) decreased from 64 (57-71) pre-surgery to 20 (16-24) at 24-month follow-up. Leg pain ( $n=9$ studies) improved from VAS 70 (65-74) pre-surgery to 17 (12-23) at 24-month interval. Disability ( $n=12$ studies), measured with the Oswestry Disability Index, decreased from 44.8 (40.1-49.4) pre-surgery to 17.3 (11.9-22.8) at 24-month follow-up. The sensitivity analysis yielded similar results.

Conclusion There is a substantial improvement in pain and disability after first-time LSF for degenerative disorders. However, long-term outcomes indicate that leg pain might be more reduced and for a longer period of time than axial back pain and disability.
\end{abstract}

Registration PROSPERO CRD42015026922.

Graphical abstract These slides can be retrieved under Electronic Supplementary Material.
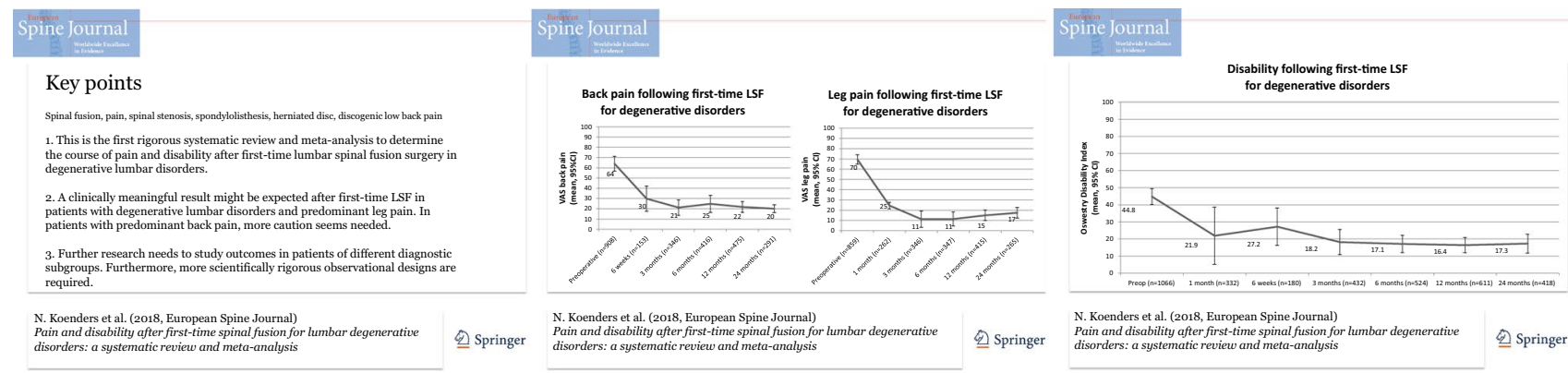

Keywords Spinal fusion $\cdot$ Pain $\cdot$ Spinal stenosis $\cdot$ Spondylolisthesis $\cdot$ Herniated disc $\cdot$ Discogenic low back pain

Electronic supplementary material The online version of this article (https://doi.org/10.1007/s00586-018-5680-3) contains supplementary material, which is available to authorized users.

Extended author information available on the last page of the article 


\section{Background}

Lumbar spinal fusion (LSF) with or without decompression surgery aims to stabilize the lumbar spine in various degenerative disorders such as spinal stenosis, spondylolisthesis, disc herniation, and discogenic low back pain [1-4]. LSF is frequently and increasingly performed [5-8] and presented in current guidelines of the North American Spine Society as 'a necessary element of the surgeon's armamentarium in the treatment of lumbar degenerative disorders' [9]. Nevertheless, LSF in degenerative disorders of the lumbar spine remains a subject of controversy. For example, outcomes in patients with chronic low back pain did not favour LSF over rehabilitation at long-term follow-up [10, 11]. In addition, two systematic reviews showed no convincing evidence of superiority of LSF compared to nonsurgical management in discogenic low back pain [3] and degenerative lumbar spondylosis [12]. Furthermore, some studies analysed costeffectiveness of LSF versus nonsurgical care in degenerative spondylolisthesis and obtained insufficient convincing evidence favouring LSF [13, 14]. Moreover, LSF should be carefully applied because of known complications such as neurologic deficit, infection, pseudarthrosis, and revision surgery [15-19]. As a result of the ongoing debate, the recent National Institute for Health and Care Excellence (NICE) guidelines recommended to not offer spinal fusion for people with low back pain unless as part of a randomized controlled trial [20].

An overview of outcomes after LSF is needed, considering that guidelines provide conflicting recommendations [9, 20] and understanding of long-term outcomes after LSF in degenerative disorders is particularly lacking [12]. Specifically, a systematic review of prospective cohort studies is crucial to understand long-term outcomes [21-23] in broad patient categories [24] and larger samples [8]. Furthermore, outcomes of prospective cohort studies are not biased by patients who might be disappointed after assignment to an undesired intervention such as 'unstructured physiotherapy' instead of LSF surgery [11]. Ultimately, the systematic review should meta-analyse the direction and tendency of outcomes after LSF surgery to improve the understanding of recovery after LSF and improve post-LSF management [25]. Therefore, the objective of this study was to systematically review and meta-analyse the course of pain (back and leg) and disability in patients with degenerative disorders of the lumbar spine, including spinal stenosis, spondylolisthesis, disc herniation, and discogenic low back pain (i.e. degenerative disc disease) after first-time LSF surgery.

\section{Methods}

\section{Protocol and registration}

The systematic review followed the methods of the predefined study protocol [26] and guidelines of the Preferred Reporting Items for Systematic Reviews and Meta-Analyses (PRISMA) statement [27]. The study was registered with PROSPERO (CRD42015026922).

\section{Eligibility criteria}

Prospective cohort studies reporting pain and disability outcomes after first-time LSF were included. The patient population involved adults (aged over 16 years) with degenerative disorders of the lumbar spine including spinal stenosis [28], spondylolisthesis [28], disc herniation [29], and discogenic low back pain (i.e. degenerative disc disease) [30]. Prospective cohort studies with consecutive patient sampling were considered most appropriate to analyse outcomes after firsttime LSF surgery as a result of a broad representation of the population [24, 31-33] and lengthy follow-up [21-23, 32, $33]$. For these reasons, randomized controlled trials were excluded [26].

\section{Search strategy, information sources, and study selection}

The search strategy was created by the first author (NK) with the support of an experienced medical librarian (RDV) and critically reviewed by all researchers [26] (Appendix 1, ESM). After approval, published studies were searched in MEDLINE, EMBASE, CINAHL, and ZETOC databases up to 31 March 2017. In addition, a search was conducted for unpublished studies (British National Bibliography for Report Literature and OpenGrey), studies in press, and studies published ahead of print. Furthermore, reference lists of included studies were checked. The language of publication was not restricted. Titles and abstracts (stage 1) followed by full texts (stage 2) were independently screened by two researchers (NK and $\mathrm{TH}$ ). In general, if there was any doubt about exclusion of the study, the study proceeded to the full-text screening stage to reduce the likelihood of excluding a relevant study. A third researcher (AR) mediated in situations where consensus could not be reached [34]. Corresponding authors were contacted by e-mail if a full text could not be retrieved. The process of study selection was summarized using a PRISMA flow diagram [27]. 


\section{Data collection and outcomes}

Data for each included study were extracted using a standardized form, which was optimized after piloting in five studies. Data were extracted independently and in duplicate by two out of three researchers (NK, AR, TH). In case of missing data, authors were contacted to provide additional information. Data were extracted on the following items: pain and disability outcomes, participants (setting and area), patient characteristics, duration of symptoms, surgical procedure(s), clinical care pathway, study design, sample size, eligibility criteria, and follow-up dates. Pain and disability outcome data were extracted at all available intervals and were measured with, for example, Visual Analogue Scale (VAS), Numeric Rating Scale (NRS), and Oswestry Disability Index (ODI) [35].

\section{Risk of bias assessment}

A modified version of the Quality in Prognostic Studies (QUIPS) tool [36, 37] was used to assess studies on the domains of representation of sample, definition of study sample, study attrition, outcome measurement, confounding, statistical analysis, provision of data, and blinding of outcomes [26] (Appendix Table 2, ESM). The overall risk of bias within a study was considered 'low' when all items were scored at 'low risk of bias' [34, 36]. Overall risk of bias was considered 'high' if one or more items within a study were scored at 'high risk of bias'. In all other cases, the overall risk of bias of a study was considered 'unclear'. Data on risk of bias were extracted independently and in duplicate by two researchers (NK, BS). Disagreements were solved by consensus in two consensus meetings. Ultimately, a third researcher (AR) mediated where consensus could not be reached. Weighted Cohen's $\kappa$ [38] was used to assess agreement between the researchers.

In addition, location bias and outcome reporting bias were assessed [34]. The presence of location bias was assumed when studies published in low or non-impact factor journals reported greater improvements than those published in high-impact journals [39]. The Spearman rank-order correlation coefficient was determined to analyse the association between impact factor and change score of disability, as the impact factors were considered of ordinal level. The change score of disability was calculated as baseline minus 1-year follow-up ODI outcome.

Information regarding impact factors of journals at year of publication was retrieved from the Journal Citation Reports database (Thomson Reuters) [40]. Outcome reporting bias was assessed by comparing outcomes listed in the study protocol or methods section with the actually reported results [34]. A modified funnel plot was constructed to investigate publication bias [34]. In the presence of publication bias, the funnel plot should resemble an asymmetrical funnel [41].

\section{Data synthesis and analysis}

A meta-analysis was conducted on pain and disability outcomes using a random effects model, maximum likelihood. Studies providing both outcome and variance data were included in the meta-analysis. As a result, an available cases analysis was performed as imputation of variance data was considered inappropriate due to a very small proportion of available variance data [34]. A sensitivity analysis with all studies was performed to assess the impact of including studies without variance data, therefore comparing $n$-weighted means with the initial meta-analysis outcomes. A visual presentation of outcomes (mean and upper bound $95 \%$ confidence interval) at all possible follow-up intervals provided optimal information to analyse the direction and tendency of outcomes. Both intervals on short- and long-term were considered valuable to determine the course of pain and disability outcomes. Both pain and disability outcomes were converted to a $0-100$ scale to facilitate comparison between studies ( 0 representing no pain or disability, 100 representing maximum pain or disability) [37]. Headrick's formula [42] was used to combine means when separate means (e.g. one-, two-, and three-level LSF surgery) described results of one study group. Individual patient data were extracted to calculate the proportion of patients who gained from LSF treatment [43] according to the minimal important change values provided by Ostelo et al. [44] (VAS 15, NRS 2, ODI $10)$. Where possible, the statistical heterogeneity of outcomes was analysed using the $I^{2}$ statistic [45].

\section{Results}

\section{Selected studies}

The search retrieved 7452 studies, a total of 5532 after removal of duplicates (Fig. 1). Following initial screening of titles and abstracts, 158 studies were considered potentially eligible. Most common reasons for exclusion on title or abstract were: other population (i.e. cervical fusion, trauma, scoliosis) or other study type (i.e. randomized controlled trial, retrospective study, or systematic review). After assessment of full text, twenty-five studies [46-70] fulfilled the eligibility criteria and were included for qualitative analysis. Boissiere et al. [47], Kok et al. [54], and Pereira et al. [60] were excluded for the quantitative analysis because of an overlap in study population with Barrey et al. [46], Kok et al. [55], and Franke et al. [68], respectively. 

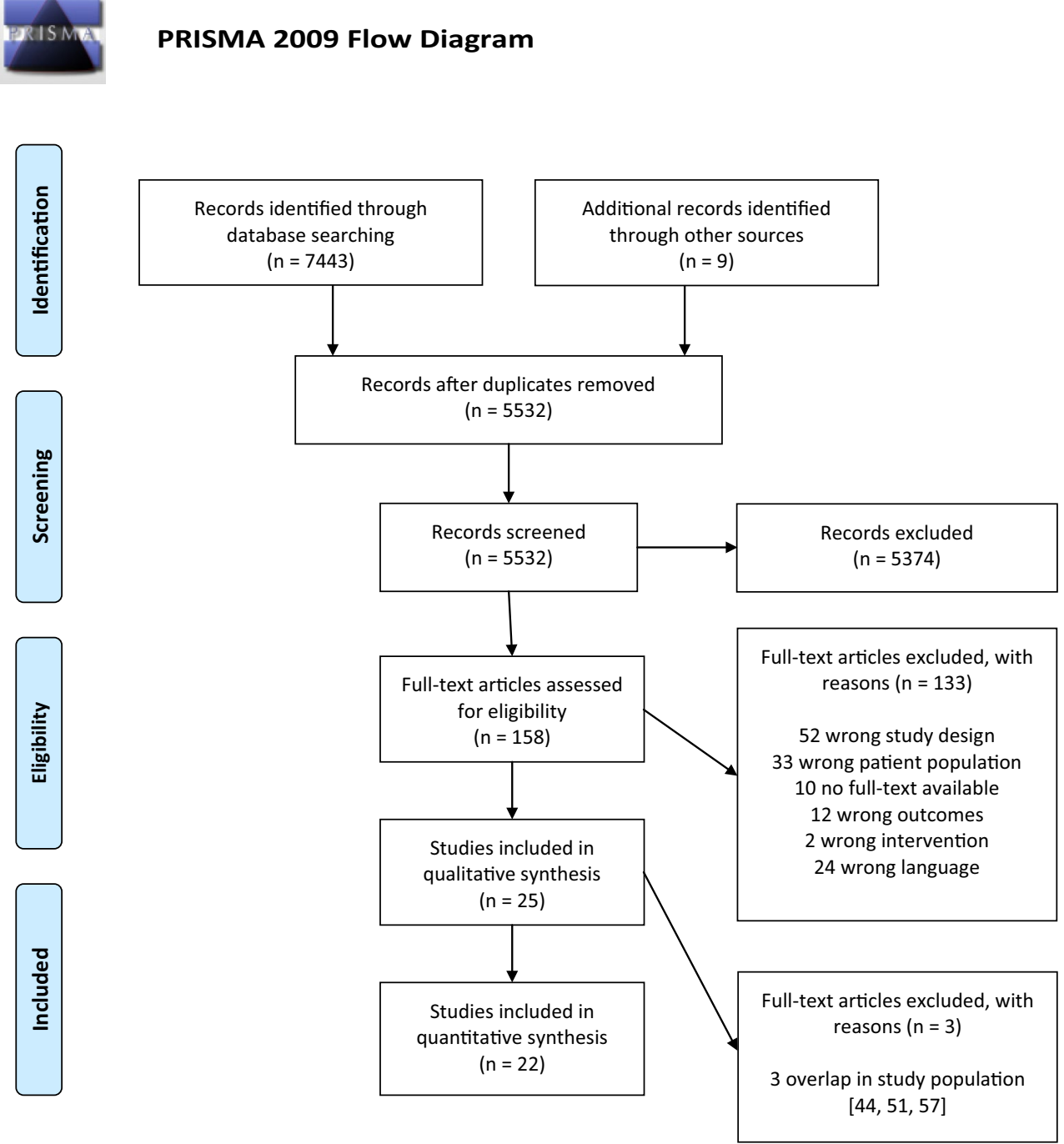

Fig. 1 Flow chart of included studies. Reproduced with permission from Moher et al. [27]

\section{Characteristics}

Tables 1 and 2 show overall study and patient characteristics, respectively. The twenty-five studies included $n=1777$ adults in total, with a range in sample size from 20 to 255 . The age of participants ranged between 17 and 87 years. The recruitment period ranged from 1996 to 2014. Pain outcomes were commonly measured with the VAS $(0-10$ or $0-100)$ for overall pain, back pain, or leg pain. Disability outcomes were measured with the ODI in all included studies except for two $[56,70]$. There was a wide variation in performed fusion techniques (Table 2). Specifically, there were different approaches (e.g. anterior or posterior), types of surgery (minimally invasive or open), types of graft (allograft and/or autograph), and cages used (yes or no). Information regarding the clinical care pathway after surgery was described in only six $[53,56-59,65]$ out of twenty-five studies.

\section{Methodological quality assessment}

In total, 163 out of 200 quality assessment items (81.5\%) were scored similarly resulting in an agreement between assessors of weighted kappa 0.61. Conclusively, 104 items were scored as low risk of bias, 88 items as unclear, and 8 items as high risk of bias (Appendix Table 2, ESM). Study attrition was scored four times as 'high risk of bias': three studies provided no information regarding loss to followup [51, 58, 59], and one study had a high loss to follow-up without proper explanation [62]. Other items were scored with high risk of bias for the following reasons: insufficient presentation of eligibility criteria and suspected inclusion of full cases only [67]; unclear selection of participants [52]; out-of-boundary outcome of VAS back pain at the preoperative follow-up interval [54]; and patient-reported pain and disability outcomes in cooperation with physician [63]. 
Table 1 Study characteristics

\begin{tabular}{|c|c|c|c|c|c|}
\hline Study (primary author) & $\begin{array}{l}\text { Recruitment } \\
\text { period (year) }\end{array}$ & Area (country) & $\begin{array}{l}\text { Centre (single } \\
\text { or multi) }\end{array}$ & $\begin{array}{l}\text { Study outcomes } \\
\text { (of interest) }\end{array}$ & Follow-up dates \\
\hline Barrey et al. [46] & 2008-2012 & France & Single & VAS, ODI & Mean $22 \operatorname{mos}( \pm 8$ mos $)$ \\
\hline Boissiere et al. [47] & 2008-2011 & France & Single & VAS, ODI & Mean $22.5 \operatorname{mos}( \pm 8.7 \mathrm{mos})$ \\
\hline Cobo Soriano et al. [48] & 2002-2006 & Spain & Single & VAS, ODI & $12 \operatorname{mos}$ \\
\hline Cuéllar et al. [66] & Not reported & USA & Multi & VAS, ODI & 2,6 weeks, $3,6,12,24 \mathrm{mos}$ \\
\hline Debnath et al. [67] & 2009-2014 & India & Single & VAS, ODI & $12 \operatorname{mos}$ \\
\hline Franke et al. [68] & 2010-2013 & EU, CAN, ME & Multi & VAS, ODI & 4 weeks, $3,6,12$ mos \\
\hline Ghogawala et al. [49] & 2010-2011 & USA & Multi & VAS, ODI & $1,3,6$, and $12 \mathrm{mos}$ \\
\hline Godil et al. [50] & 2009-2010 & USA & Single & NRS, ODI & $24 \operatorname{mos}$ \\
\hline Hagenmaier et al. [51] & 2010-2012 & NL & N.r. & VAS, ODI & 6 weeks, $3,6,12$ mos \\
\hline Inage et al. [52] & Not reported & Japan & Single & VAS, ODI & $24 \operatorname{mos}$ \\
\hline Kanter et al. [69] & 2009-2013 & USA & Multi & VAS, ODI & 6 weeks, $6,12,24 \mathrm{mos}$ \\
\hline Kleeman et al. [53] & $1998-2000$ & USA & Single & VAS, ODI & $6,12 \mathrm{mos}$ \\
\hline Kok et al. [54] & 2004-2006 & NL & Single & ODI & $1,3,6,12,24 \operatorname{mos}$ \\
\hline Kok et al. [55] & Not reported & $\mathrm{UK}, \mathrm{NL}$ & Multi & VAS, ODI & $1,3,6,12,24 \operatorname{mos}$ \\
\hline Lara-Almunia et al. [56] & $2001-2010$ & Spain & Single & VAS & $3,6,12 \mathrm{mos}$ \\
\hline Lee et al. [57] & Not reported & Korea & N.r. & VAS, ODI & 6 weeks, $3,6,12,18,24$ mos \\
\hline Mao et al. [58] & 2006-2010 & China & Single & VAS, ODI & Immediate, $3,6,12,24,36 \mathrm{mos}$ \\
\hline Pavlov et al. [59] & 1996-1998 & NL & N.r. & VAS, ODI & 6 weeks, $3,6,12,24,48 \mathrm{mos}$ \\
\hline Pereira et al. [60] & 2010-2012 & EU, CAN, ME & Multi & VAS, ODI & 4 weeks \\
\hline Pimenta et al. [61] & 2005-2006 & Brazil & Single & VAS, ODI & $1,3,6,12 \mathrm{mos}$ \\
\hline Rhee et al. [70] & 2009-2011 & USA & Single & VAS & $12 \operatorname{mos}$ \\
\hline Tobler and Ferrara [62] & 2005-2006 & USA & N.r. & VAS, ODI & 3 weeks, $3,6,12,24$ mos \\
\hline Trouillier et al. [63] & $1997-2000$ & Germany & Single & VAS, ODI & $3,6,12,42 \operatorname{mos}$ \\
\hline Tsahtsarlis and Wood [64] & 2008-2010 & Australia & Single & ODI & $6 \mathrm{mos}$ \\
\hline $\mathrm{Xu}$ et al. [65] & 2001-2006 & China & Single & ODI & $3,6,12,24 \operatorname{mos}$ \\
\hline
\end{tabular}

CAN Canada, EU Europe, $M E$ Middle East, mos months, $N L$ The Netherlands, N.r. not reported, ODI Oswestry Disability Index, UK United Kingdom, USA United States of America, VAS Visual Analogue Scale

Ultimately, 17 studies were considered at unclear risk of bias and eight with high risk of bias.

\section{Location bias}

Analysis of indexing of studies shows that all studies were indexed in MEDLINE (Appendix Table 3, ESM). Fourteen studies [46-50, 53, 59-61, 63, 64, 66, 69, 70] were indexed in EMBASE and two studies [51, 53] in CINAHL. The impact factors of the journals ranged from 0.430 to 5.163 [40] (Appendix Table 3, ESM). Correlation analysis between rank of impact factor and rank of change score resulted in a (negative) Spearman's rho coefficient of -0.266 . Analysis of indexing did not seem to show signs of location bias.

\section{Outcome reporting bias}

Only four studies $[49,60,68,69]$ reported use of a published study protocol. Ghogawala et al. [49], Franke et al. [68], and Kanter et al. [69] provided data at all follow-up intervals and thorough description of their primary and secondary outcome selection. Pereira et al. [60] described in their study protocol measurement of VAS (back pain and leg pain) and ODI at baseline, 4 weeks and 3,6, and 12 months, but outcomes at 3-, 6-, and 12-month follow-up were not reported.

\section{Publication bias}

The modified funnel plot to assess possible publication bias did not indicate selective publication of disability outcomes in relation to the study sample size (Fig. 2). 


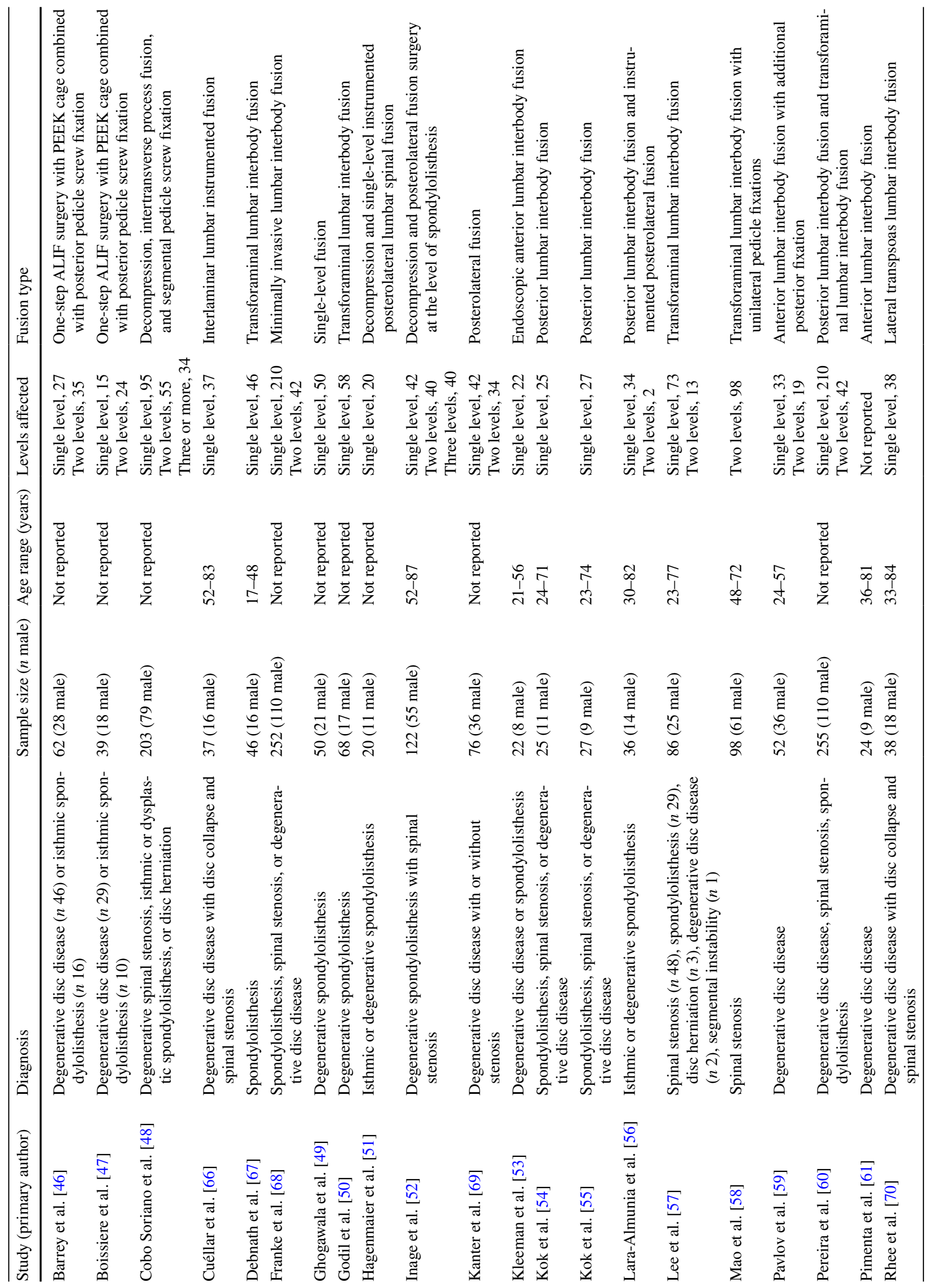




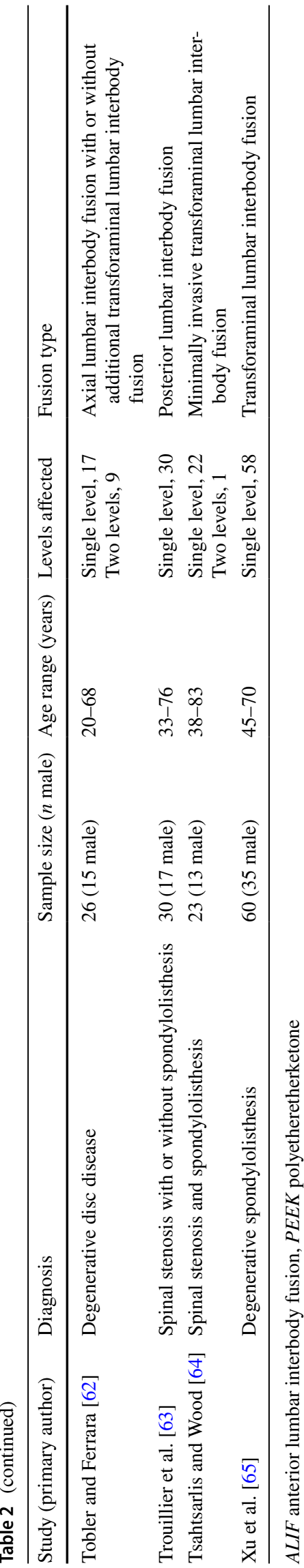

\section{Pain outcomes}

Four cohorts reported pain outcomes and variance data on a VAS scale for both back and leg pain. Nine studies described VAS back pain outcomes, and nine studies reported outcomes with the VAS leg pain. One study analysed pain outcomes with the back pain NRS and leg pain NRS [50]. Three studies described pain outcomes for subgroups. Cobo Soriano et al. [48] created a 'disc herniation' and 'other lumbar spine disorders' group. Inage et al. [52] made three groups: one-level fusion, two-level fusion, and three-level fusion. Pavlov et al. [59] created a 'single-level' and 'double-level' groups. When converted to a 100-point scale, mean preoperative outcomes of individual studies ranged from VAS back and leg pain 70-93, VAS back pain 44-79, and VAS leg pain 57-82.4. The course of pain following first-time LSF for degenerative disorders at different follow-up intervals is presented in Fig. 3a, b, c. Details of the meta-analysis of pain outcomes are provided in Appendix 4 (ESM).

\section{Disability outcomes}

Twelve cohorts included in the quantitative synthesis reported disability outcomes using ODI scores. Cobo Soriano et al. [48], Inage et al. [52], and Pavlov et al. [59] created subgroups similar to the reporting of pain outcomes. The disability outcomes at the preoperative assessment point ranged from 24.5 to 62.8 . The course of disability following first-time LSF for degenerative disorders at different follow-up intervals is shown in Fig. 4. Details of the meta-analysis of disability outcomes are provided in Appendix 4 (ESM).

\section{Sensitivity analysis}

The sensitivity analysis, including studies without variance data, included data of two additional cohorts on VAS back and leg pain outcomes, three cohorts on VAS back pain, three cohorts on VAS leg pain, and eight cohorts on disability. Pain and disability outcomes showed similar means and courses in studies with and without variance data. However, the sensitivity analysis added data on very long-term followup ( $\geq 42$ months). An increase in back pain and disability outcomes was considered notable. Details of the sensitivity analysis of pain and disability outcomes are provided in Appendix 4 (ESM).

\section{Subgroup analysis}

A meta-analysis of outcomes for different diagnostic subgroups was not possible with the available data. Fifteen 
Fig. 2 Funnel plot presenting sample size versus change score of disability outcomes to assess publication bias. $n$ number

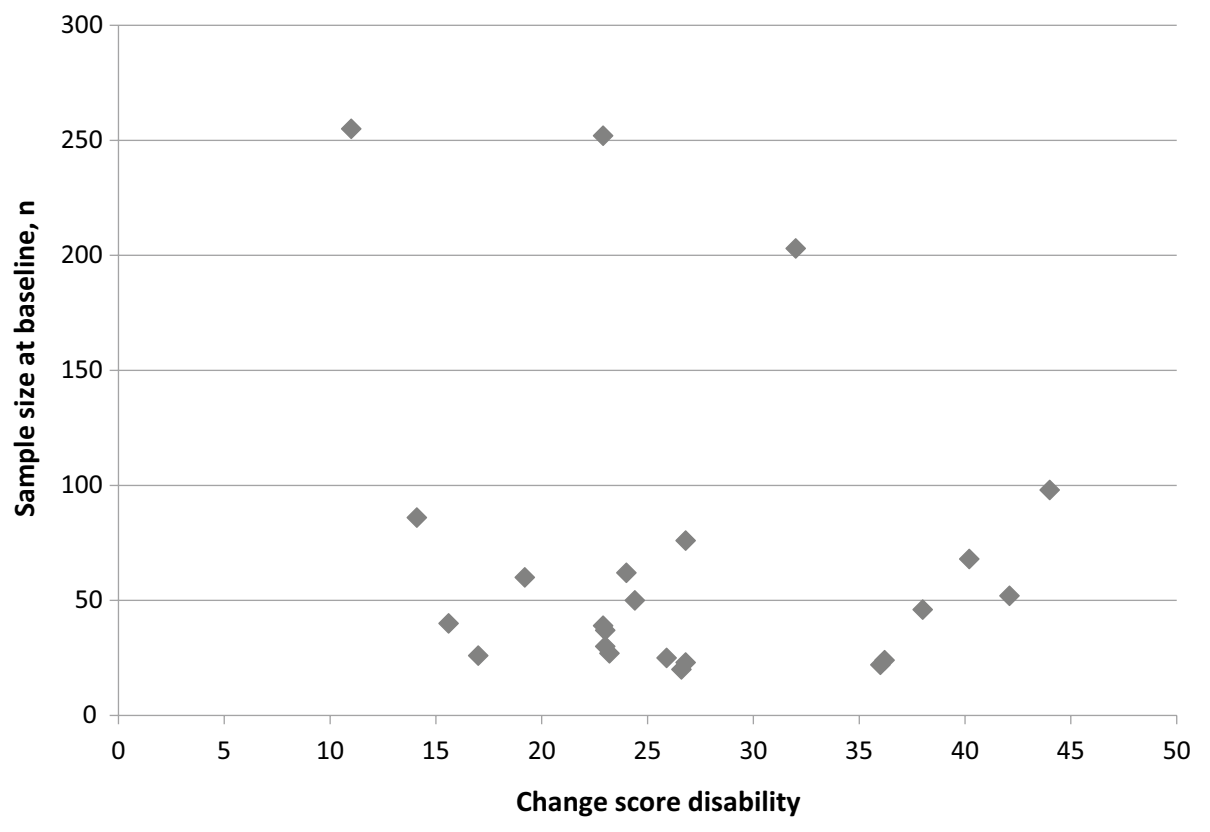

studies presented blended data of different diagnostic subgroups without presenting outcomes per diagnostic subgroup. The remaining ten studies reported outcomes for eleven diagnostic subgroups: 1 spinal stenosis, 6 spondylolisthesis, 1 disc herniation, 0 discogenic low back pain, and 3 degenerative disc disease. Unfortunately, there were no follow-up intervals presented by two studies within one diagnostic subgroup and authors could not be reached to provide additional data, making a subgroup analysis impossible.

Nevertheless, two studies [52, 59] performed a subgroup analysis based on different numbers of fused levels. Both studies did not find a statistical significant difference in pain and disability outcomes and number of affected levels. Leg pain before surgery was reported in thirteen studies [48, 50-52, 55-57, 60, 63, 66-68, 70]. However, these studies did not provide data regarding an association between leg pain before and after surgery. Three studies $[48,51,53]$ in total included 75 participants smoking and 148 not smoking. Other studies did not provide information regarding smoking or did not provide amounts of non-smokers. Furthermore, three studies reported work status of their participants. Ghogawala et al. [49] reported that 24 out of 50 patients were working preoperatively, similar to a 13 out of 18 and 79 out of 143 in the studies of, respectively, Kleeman et al. [53] and Franke et al. [68]. Neither of the studies performed subgroup analysis nor provided information regarding type of work and income. One study [50] identified 17 out of 58 patients with depression at time of surgery. Pain catastrophizing was not reported in a single study.

\section{Complications}

A variety of complications following LSF were reported (Table 3) [46, 47, 49, 53-60, 62-65, 68, 69], including: reoperation, cage migration, cage breakage, malposition of pedicle screw, wound infection, adjacent segment instability, pseudarthrosis, dural tear, spinal haematoma, vascular wound, aortic occlusion with non-fatal cardiac arrest, myocardial infarction, pulmonary embolism, acute allergic reaction, urosepsis, bowel injury, and postoperative confusion. There were no cases of surgery-related mortality reported.

\section{Discussion}

This is the first rigorous systematic review and meta-analysis to determine the course of pain and disability after first-time LSF surgery in degenerative disorders. In summary, back and leg pain outcomes showed a decrease at every follow-up interval compared to preoperative levels. Back pain decreased at several times of follow-up ranging between 20 and 30 (respective upper bounds 95\% confidence interval, 24 and 42). However, sensitivity analysis showed an increase to an $n$-weighted mean VAS back pain of 45 at 42-month follow-up in one study at high risk of bias [63]. In contrast, leg pain improved substantially at both short- and long-term follow-up. The decrease in leg pain was more distinct due to a seriously decreased leg pain at the 1-and 3-month intervals, which might be a result of successful nerve root decompression after LSF [71-74]. 
Fig. 3 a VAS back pain and leg pain, $\mathbf{b}$ course of back pain, $\mathbf{c}$ course of leg pain (mean and upper bound $95 \% \mathrm{CI}$ ) following first-time LSF for degenerative disorders. $C I$ confidence interval, $L S F$ lumbar spinal fusion, $n$ number of patients, VAS Visual Analogue Scale
Pain following first-time LSF

for degenerative disorders

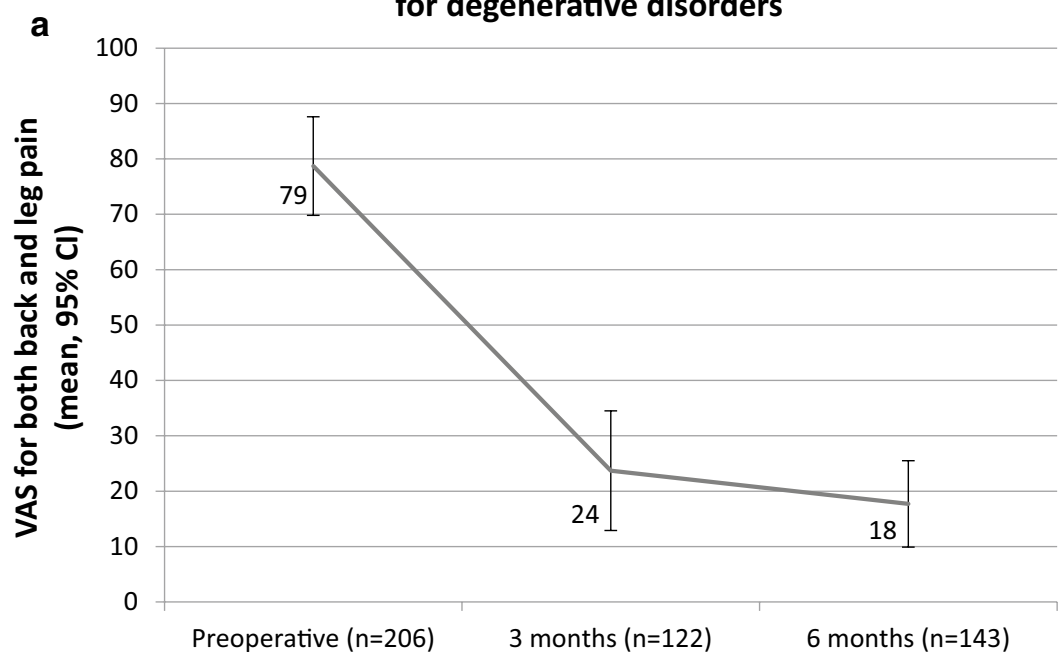

Back pain following first-time LSF

for degenerative disorders
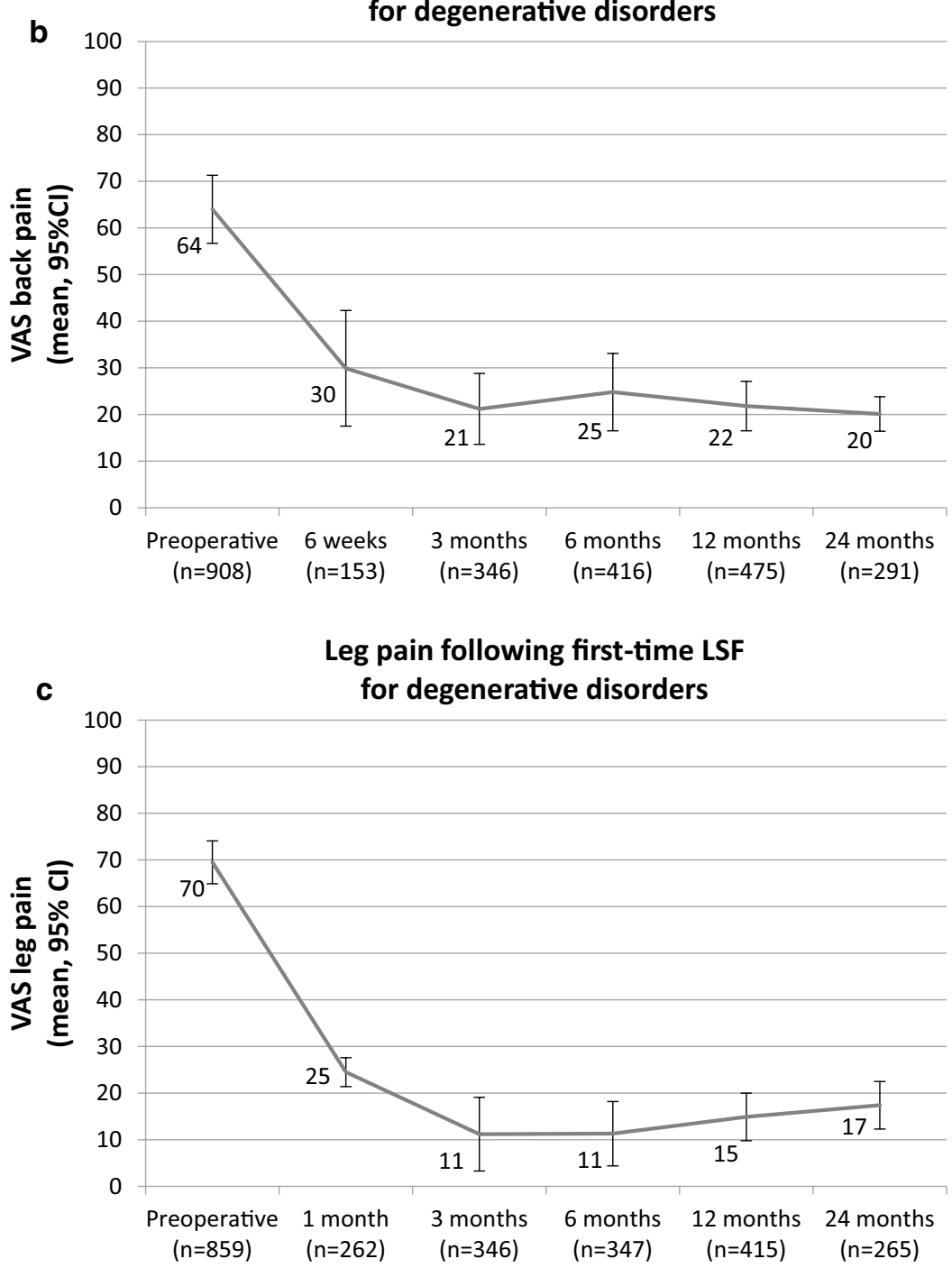
Fig. 4 Course of disability (mean and upper bound 95\% CI) following first-time LSF for degenerative disorders. $C I$ confidence interval, $L S F$ lumber spinal fusion, $n$ number of patients, preop preoperative

\section{Disability following first-time LSF for degenerative disorders}

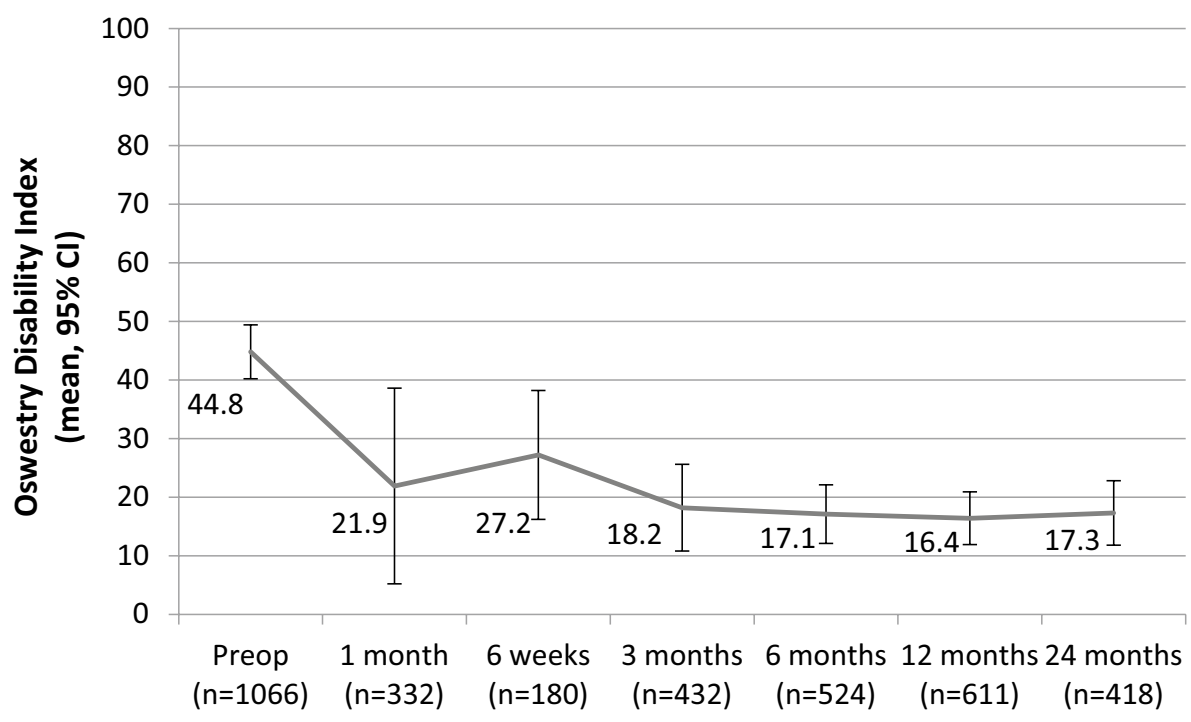

Furthermore, the mean leg pain outcomes seem lower at all follow-up intervals compared to the back pain outcomes. The severity of disability showed a relatively steady decrease over time, with an exception of the 6-week interval $[57,61,69]$. In addition, sensitivity analysis showed an increase to an $n$-weighted mean ODI of 30 and 24.6 at the 42-month [63] and 48-month follow-up [59], taking into account both studies at high risk of bias. In light of the a priori $[26,44]$ formulated minimal important change values, the course after first-time LSF surgery in degenerative disorders showed an overall clinically relevant decrease in pain and disability outcomes. A clinically significant decrease in back pain and disability at long-term follow-up might be questionable. Nevertheless, the long-term results should be interpreted with caution as a result of risk of bias and lacking variance data.

The findings were highly comparable with results presented in the systematic review and meta-analysis of randomized controlled trials and observational studies by Phan et al. [75], which compared back pain and disability outcomes between minimally invasive and open transforaminal lumbar interbody fusion in treatment of degenerative lumbar disease. Analysis comparing severity of back pain (VAS) and disability (ODI) at last follow-up showed an $n$-weighted mean of, respectively, 25.4 and 16.2 , quite similar to the 20 and 16.4 at 24-month follow-up presented in the current study. Carreon et al. [76] reported similar disability (ODI) outcomes at a minimum of 1-year follow-up of ( $n$-weighted mean) 28.3 for the overall surgical population in studies comparing LSF and nonsurgical interventions in various degenerative lumbar spine disorders.

\section{Strengths and limitations}

This is the first systematic review and meta-analysis analysing the long-term course of pain and disability following LSF. The knowledge gained can help guide treatment decision-making to improve patient selection (those with predominant leg pain) and decrease post-surgery back pain. The current course of back pain might indicate a need for improved preoperative selection, medical treatment, and physiotherapy management. The current quantitative analysis included twenty-two studies, representing a large sample of 1777 adults with degenerative disorders. Moreover, the availability of numerous cohorts is likely to improve generalizability of our results and support our decision to forgo the inclusion of randomized controlled trials, which might be biased by the utilization of (too) strict eligibility criteria [22, 24, 25]. Finally, the use of a thoroughly developed and published study protocol [26] has improved reproducibility and validity of the findings.

Nonetheless, a few deviations from the study protocol had to be made. For example, variance data were not available in seven studies [48-50, 59, 62, 63, 67] which decreased the power and generalizability of the metaanalysis outcomes. A sensitivity analysis with $n$-weighted means was performed to assess the impact of including studies without variance data and indicated no difference of outcomes. In addition, diagnostic heterogeneity of included patients is likely to influence the pain and disability outcomes, as patient-reported outcomes after LSF might be dependent on the clinical diagnosis $[77,78]$. The patients included in the current study do not equally represent all 
Table 3 Overview of surgeryrelated complications

\begin{tabular}{|c|c|c|c|}
\hline Study (primary author) & Sample size & Complication(s), $n$ & $\begin{array}{l}\text { Revision } \\
\text { surgery, } n\end{array}$ \\
\hline Barrey et al. [46] & 62 & Vascular wound, 1 & No \\
\hline Boissiere et al. [47] & 39 & Vascular wound, 1 & No \\
\hline Franke et al. [68] & 252 & $\begin{array}{l}\text { Adverse events, } 50 \\
\text { Serious adverse events, } 9 \text { (out of } 50 \text { ) }\end{array}$ & No \\
\hline Ghogawala et al. [49] & 50 & $\begin{array}{l}\text { Aortic occlusion with non-fatal cardiac arrest, } 1 \\
\text { CSF leak, } 1 \\
\text { Instrumentation issue, } 1\end{array}$ & Yes, 1 \\
\hline Kanter et al. [69] & 76 & $\begin{array}{l}\text { Cage migration, } 1 \\
\text { Nerve root impingement, } 1 \\
\text { Pedicle screw disconnected, } 1 \\
\text { Wound infection, } 1\end{array}$ & Yes, 3 \\
\hline Kleeman et al. [53] & 22 & $\begin{array}{l}\text { Bowel injury, } 1 \\
\text { Vascular injury, } 1\end{array}$ & No \\
\hline Kok et al. [54] & 25 & $\begin{array}{l}\text { Cage migration, } 2 \\
\text { Dural tear, } 4 \\
\text { Myocardial infarction, } 1\end{array}$ & Yes, 2 \\
\hline Kok et al. [55] & 27 & $\begin{array}{l}\text { Cage breakage, } 1 \\
\text { Dural tear, } 2 \\
\text { Pedicle screw malposition, } 1\end{array}$ & N.r. \\
\hline Lara-Almunia et al. [56] & 36 & $\begin{array}{l}\text { Cerebrospinal fluid fistula, } 1 \\
\text { Neurological deficit (transient), } 2 \\
\text { Wound infection, } 2\end{array}$ & Yes, 1 \\
\hline Lee et al. [57] & 86 & $\begin{array}{l}\text { Pedicle screw malposition, } 2 \\
\text { Pseudarthrosis, } 4 \\
\text { Wound infection, } 2\end{array}$ & Yes, 1 \\
\hline Mao et al. [58] & 98 & Intervertebral disc infection, 1 & Yes, 1 \\
\hline Pavlov et al. [59] & 52 & $\begin{array}{l}\text { Pedicle crew breakage, } 1 \\
\text { Pedicle screw malposition, } 2 \\
\text { Stenosis (new), } 1 \\
\text { Wound infection, } 3\end{array}$ & Yes, 5 \\
\hline Pereira et al. [60] & 255 & $\begin{array}{l}\text { Adverse events, } 39 \\
\text { Serious adverse events, } 6\end{array}$ & Yes, 1 \\
\hline Tobler and Ferrara [62] & 26 & $\begin{array}{l}\text { Adverse events, } 34 \\
\text { Serious adverse events, } 0\end{array}$ & No \\
\hline Trouillier et al. [63] & 30 & $\begin{array}{l}\text { Adjacent segment instability, } 1 \\
\text { Instrumentation loosening, } 1 \\
\text { Nerve root compression (persistent), } 1 \\
\text { Pleural effusion (transient), } 1 \\
\text { Pulmonary embolism, } 1 \\
\text { Sacroiliac joint pain, } 4\end{array}$ & Yes, 1 \\
\hline Tsahtsarlis and Wood [64] & 23 & $\begin{array}{l}\text { Nerve root compression (transient), } 1 \\
\text { Pedicle screw malposition, } 1 \\
\text { Pulmonary embolism, } 1\end{array}$ & No \\
\hline Xu et al. [65] & 60 & $\begin{array}{l}\text { Dural tear, } 2 \\
\text { Nerve root compression (persistent), } 2 \\
\text { Neurological deficit (transient), } 1 \\
\text { Pedicle screw migration, } 1 \\
\text { Pseudarthrosis, } 1\end{array}$ & Yes, 4 \\
\hline
\end{tabular}

$n$ number

diagnostic subgroups (spinal stenosis, spondylolisthesis, disc herniation, discogenic low back pain), and therefore it remains unclear whether it is valid to generalize the results to all subgroups of patients with degenerative disorders of the lumbar spine.

\section{Recommendations for future research}

Future research is needed to improve understanding of the course of pain and disability in patients of different diagnostic subgroups and different back pain trajectories 
$[79,80]$. A large rigid cohort with broad patient categories would make precise subgroup analyses possible. In addition, research should provide more information regarding the clinical care pathway, psychosocial and physical conditions of the patients, medical treatment, and physiotherapy on both short- and long-term. Without this information, it remains impossible to improve LSF management. Furthermore, future research should focus on systematically collecting performance data to augment the patient-reported outcomes [81]. To end, it seems necessary to concentrate research in patients after first-time lumbar fusion for degenerative lumbar disorders on very long-term follow-up intervals ( $\geq 42$ months), as a part of the post-surgery improvement in outcomes seems to decline.

\section{Conclusion}

Overall, both pain and disability outcomes improved after first-time lumbar spinal fusion for degenerative disorders. Results of the current study indicate that leg pain might be more reduced and for a longer period of time than axial back pain and disability. In patients with predominant back pain, more caution seems needed. In conclusion, a clinically meaningful result might be expected after first-time lumbar spinal fusion in patients with degenerative lumbar disorders and predominant leg pain.

Acknowledgements We acknowledge Rikie Deurenberg-Vos (RDV), information specialist at the Radboud University Medical Center, for her contribution to the search strategy.

\section{Compliance with ethical standards}

Conflict of interest All authors declare that they have no conflict of interest.

Open Access This article is distributed under the terms of the Creative Commons Attribution 4.0 International License (http://creativeco mmons.org/licenses/by/4.0/), which permits unrestricted use, distribution, and reproduction in any medium, provided you give appropriate credit to the original author(s) and the source, provide a link to the Creative Commons license, and indicate if changes were made.

\section{References}

1. Wang JC et al (2014) Guideline update for the performance of fusion procedures for degenerative disease of the lumbar spine. Part 8: lumbar fusion for disc herniation and radiculopathy. J Neurosurg Spine 21(1):48-53

2. Resnick DK et al (2014) Guideline update for the performance of fusion procedures for degenerative disease of the lumbar spine. Part 9: lumbar fusion for stenosis with spondylolisthesis. J Neurosurg Spine 21(1):54-61
3. Bydon M et al (2014) Lumbar fusion versus nonoperative management for treatment of discogenic low back pain: a systematic review and meta-analysis of randomized controlled trials. J Spinal Disord Tech 27(5):297-304

4. Resnick DK et al (2014) Guideline update for the performance of fusion procedures for degenerative disease of the lumbar spine. Part 10: lumbar fusion for stenosis without spondylolisthesis. J Neurosurg Spine 21(1):62-66

5. Kalakoti $P$ et al (2015) Inpatient outcomes and post-operative complications following primary versus revision lumbar spinal fusion surgeries for degenerative lumbar disc disease: a national (nationwide) inpatient sample analysis 2002-2011. World Neurosurg 85:114-124

6. Rajaee SS et al (2012) Spinal fusion in the United States: analysis of trends from 1998 to 2008. Spine (Phila Pa 1976) 37(1):67-76

7. Hart R et al (2015) Quality and quantity of published studies evaluating lumbar fusion during the past 10 years: a systematic review. Glob spine J 28(03):207-218

8. Machado GC et al (2017) Trends, complications, and costs for hospital admission and surgery for lumbar spinal stenosis. Spine (Phila Pa 1976) 22:1737-1743

9. Kaiser MG et al (2014) Guideline update for the performance of fusion procedures for degenerative disease of the lumbar spine. Part 1: introduction and methodology. J Neurosurg Spine 21(1):2-6

10. Mannion AF, Brox JI, Fairbank JC (2013) Comparison of spinal fusion and nonoperative treatment in patients with chronic low back pain: long-term follow-up of three randomized controlled trials. Spine J 13(11):1438-1448

11. Mannion AF, Brox JI, Fairbank JC (2016) Consensus at last! Long-term results of all randomized controlled trials show that fusion is no better than non-operative care in improving pain and disability in chronic low back pain. Spine J 16(5):588-590

12. Gibson JN, Waddell G (2005) Surgery for degenerative lumbar spondylosis: updated Cochrane Review. Spine (Phila Pa 1976) 30(20):2312-2320

13. Mummaneni PV et al (2014) Cost-effectiveness of lumbar discectomy and single-level fusion for spondylolisthesis: experience with the NeuroPoint-SD registry. Neurosurg Focus 36(6):E3

14. Tosteson AN et al (2011) Comparative effectiveness evidence from the spine patient outcomes research trial: surgical versus nonoperative care for spinal stenosis, degenerative spondylolisthesis, and intervertebral disc herniation. Spine (Phila Pa 1976) 36(24):2061-2068

15. Rihn JA et al (2009) Complications associated with single-level transforaminal lumbar interbody fusion. Spine J 9(8):623-629

16. Carragee EJ, Hurwitz EL, Weiner BK (2011) A critical review of recombinant human bone morphogenetic protein-2 trials in spinal surgery: emerging safety concerns and lessons learned. Spine J 11(6):471-491

17. Jorgenson SS et al (1994) A prospective analysis of autograft versus allograft in posterolateral lumbar fusion in the same patient. A minimum of 1-year follow-up in 144 patients. Spine (Phila Pa 1976) 19(18):2048-2053

18. Dimar JR 2nd et al (2009) Clinical and radiographic analysis of an optimized rhBMP-2 formulation as an autograft replacement in posterolateral lumbar spine arthrodesis. J Bone Jt Surg Am 91(6): 1377-1386

19. Burkus JK et al (2011) Blood serum antibody analysis and longterm follow-up of patients treated with recombinant human bone morphogenetic protein-2 in the lumbar spine. Spine (Phila $\mathrm{Pa}$ 1976) 36(25):2158-2167

20. The National Guideline Centre (UK) (2016) Low back pain and sciatica in over 16s: assessment and management. 2016, NICE, London 
21. Faraoni D, Schaefer ST (2016) Randomized controlled trials versus observational studies: why not just live together? BMC Anesthesiol 16(1):102

22. Jadad AR, Enkin M (2007) Randomized controlled trials: questions, answers, and musings. Wiley Online Library, New York

23. Benson K, Hartz AJ (2000) A comparison of observational studies and randomized, controlled trials. N Engl J Med 342(25):1878-1886

24. Iorio A et al (2015) Use of GRADE for assessment of evidence about prognosis: rating confidence in estimates of event rates in broad categories of patients. BMJ 350:h870

25. Costa LDCM et al (2012) The prognosis of acute and persistent low-back pain: a meta-analysis. Can Med Assoc J. https://doi. org/10.1503/cmaj.111271

26. Koenders $\mathrm{N}$ et al (2016) Pain and disability following first-time lumbar fusion surgery for degenerative disorders: a systematic review protocol. Syst Rev 5:72

27. Moher D et al (2009) Preferred reporting items for systematic reviews and meta-analyses: the PRISMA statement. Ann Intern Med 151(4):264-269

28. Kreiner DS et al (2013) An evidence-based clinical guideline for the diagnosis and treatment of degenerative lumbar spinal stenosis (update). Spine J 13(7):734-743

29. Kreiner DS et al (2014) An evidence-based clinical guideline for the diagnosis and treatment of lumbar disc herniation with radiculopathy. Spine J 14(1):180-191

30. Chou $\mathrm{R}$ et al (2007) Diagnosis and treatment of low back pain: a joint clinical practice guideline from the American College of Physicians and the American Pain Society. Ann Intern Med 147(7):478-491

31. Bothwell LE et al (2016) Assessing the gold standard-lessons from the history of RCTs. N Engl J Med 374(22):2175-2181

32. Chavez-MacGregor M, Giordano SH (2016) Randomized clinical trials and observational studies: is there a battle? J Clin Oncol 34(8):772-773

33. Concato J, Shah N, Horwitz RI (2000) Randomized, controlled trials, observational studies, and the hierarchy of research designs. N Engl J Med 342(25):1887-1892

34. Higgins J, Green S (2011) Cochrane handbook for systematic reviews of interventions version 5.1. 0 [updated March 2011]. The Cochrane Collaboration. www.cochrane-handbook.org

35. Ghogawala $\mathrm{Z}$ et al (2014) Guideline update for the performance of fusion procedures for degenerative disease of the lumbar spine. Part 2: assessment of functional outcome following lumbar fusion. J Neurosurg Spine 21(1):7-13

36. Hayden JA et al (2013) Assessing bias in studies of prognostic factors. Ann Intern Med 158(4):280-286

37. Pengel LH et al (2003) Acute low back pain: systematic review of its prognosis. BMJ 327(7410):323

38. Cohen J (1968) Weighted kappa: nominal scale agreement provision for scaled disagreement or partial credit. Psychol Bull 70(4):213

39. Pittler $\mathrm{M}$ et al (2000) Location bias in controlled clinical trials of complementary/alternative therapies. J Clin Epidemiol 53(5):485-489

40. Reuters T (2017) InCites. see https://jcr.incites.thomsonreuters. com/JCRJournalHomeAction.action. Accessed 10 April 2017

41. Egger M et al (1997) Bias in meta-analysis detected by a simple, graphical test. BMJ 315(7109):629-634

42. Headrick TC (2009) Statistical simulation: power method polynomials and other transformations. CRC Press, Boca Raton

43. Guyatt $\mathrm{GH}$ et al (1998) Interpreting treatment effects in randomised trials. BMJ 316(7132):690-693

44. Ostelo RW et al (2008) Interpreting change scores for pain and functional status in low back pain: towards international consensus regarding minimal important change. Spine (Phila Pa 1976) 33(1):90-94

45. Higgins JP et al (2003) Measuring inconsistency in meta-analyses. BMJ 327(7414):557-560

46. Barrey CY et al (2013) One-stage combined lumbo-sacral fusion, by anterior then posterior approach: clinical and radiological results. Eur Spine J 22(Suppl 6):S957-S964

47. Boissiere L et al (2013) Lumbar-sacral fusion by a combined approach using interbody PEEK cage and posterior pediclescrew fixation: clinical and radiological results from a prospective study. Orthop Traumatol Surg Res 99(8):945-951

48. Cobo Soriano J et al (2010) Predictors of outcome after decompressive lumbar surgery and instrumented posterolateral fusion. Eur Spine J 19(11):1841-1848

49. Ghogawala $\mathrm{Z}$ et al (2013) The efficacy of lumbar discectomy and single-level fusion for spondylolisthesis: results from the NeuroPoint-SD registry: clinical article. J Neurosurg Spine 19(5):555-563

50. Godil SS et al (2014) Accurately measuring the quality and effectiveness of lumbar surgery in registry efforts: determining the most valid and responsive instruments. Spine $\mathbf{J}$ 14(12):2885-2891

51. Hagenmaier F et al (2013) Changes in bone mineral density in the intertransverse fusion mass after instrumented single-level lumbar fusion: a prospective 1-year follow-up. Spine (Phila Pa 1976) 38(8):696-702

52. Inage $\mathrm{K}$ et al (2011) One, two-, and three-level instrumented posterolateral fusion of the lumbar spine with a local bone graft: a prospective study with a 2-year follow-up. Spine (Phila Pa 1976) 36(17):1392-1396

53. Kleeman TJ, Ahn UM, Talbot-Kleeman A (2001) Laparoscopic anterior lumbar interbody fusion with rhBMP-2: a prospective study of clinical and radiographic outcomes. Spine (Phila Pa 1976) 26(24):2751-2756

54. Kok D et al (2012) The memory metal minimal access cage: a new concept in lumbar interbody fusion-a prospective, noncomparative study to evaluate the safety and performance. Adv Orthop 2012:898606

55. Kok D et al (2012) The memory metal spinal system in a posterior lumbar interbody fusion (PLIF) procedure: a prospective, non-comparative study to evaluate the safety and performance. Open Orthop J 6:220-225

56. Lara-Almunia M, Gomez-Moreta JA, Hernandez-Vicente J (2015) Posterior lumbar interbody fusion with instrumented posterolateral fusion in adult spondylolisthesis: description and association of clinico-surgical variables with prognosis in a series of 36 cases. Int J Spine Surg 9:22

57. Lee JC, Jang HD, Shin BJ (2012) Learning curve and clinical outcomes of minimally invasive transforaminal lumbar interbody fusion: our experience in 86 consecutive cases. Spine (Phila Pa 1976) 37(18):1548-1557

58. Mao L et al (2014) Bilateral decompression using a unilateral pedicle construct for lumbar stenosis. Int Orthop 38(3):573-578

59. Pavlov PW et al (2004) Good outcome and restoration of lordosis after anterior lumbar interbody fusion with additional posterior fixation. Spine (Phila Pa 1976) 29(17):1893-1899 (discussion 1900)

60. Pereira P et al (2015) Surgical data and early postoperative outcomes after minimally invasive lumbar interbody fusion: results of a prospective, multicenter, observational data-monitored study. PLoS ONE 10(3):e0122312

61. Pimenta L, Pesantez CF, Oliveira L (2008) Silicon matrix calcium phosphate as a bone substitute: early clinical and radiological results in a prospective study with 12-month follow-up. SAS J Electron Resour 2(2):62-68 
62. Tobler WD, Ferrara LA (2011) The presacral retroperitoneal approach for axial lumbar interbody fusion: a prospective study of clinical outcomes, complications and fusion rates at a followup of 2 years in 26 patients. J Bone Jt Surg Br 93(7):955-960

63. Trouillier H et al (2006) Posterior lumbar interbody fusion (PLIF) with cages and local bone graft in the treatment of spinal stenosis. Acta Orthop Belg 72(4):460-466

64. Tsahtsarlis A, Wood M (2012) Minimally invasive transforaminal lumbar interbody fusion and spondylolisthesis. J Clin Neurosci 19(6):858-861

65. Xu H, Tang H, Li Z (2009) Surgical treatment of adult degenerative spondylolisthesis by instrumented transforaminal lumbar interbody fusion in the Han nationality. J Neurosurg Spine 10(5):496-499

66. Cuellar JM, Field JS, Bae HW (2016) Distraction laminoplasty with interlaminar lumbar instrumented fusion (ILIF) for lumbar stenosis with or without grade 1 spondylolisthesis: technique and 2-year outcomes. Spine 41(Suppl 8):S97-S105

67. Debnath UK et al (2016) Interbody fusion in low grade lumbar spondylolsithesis: clinical outcome does not correalte with slip reduction and neural foraminal dimension. Asian Spine J 10(2):314-320

68. Franke J et al (2016) MASTERS-d study: a prospective, multicenter, pragmatic, observational, data-monitored trial of minimally invasive fusion to treat degenerative lumbar disorders, 1-year follow-up. Cureus 8(6):e640

69. Kanter AS et al (2016) A prospective, multi-center clinical and radiographic outcomes evaluation of ChronOS strip for lumbar spine fusion. J Clin Neurosci 25:36-40

70. Rhee JW et al (2015) Prospective evaluation of 1-year outcomes in single-level percutaneous lumbar transfacet screw fixation in the lateral decubitus position following lateral transpsoas interbody fusion. Eur Spine J 24(11):2546-2554

71. Rao PJ et al (2015) Stand-alone anterior lumbar interbody fusion for treatment of degenerative spondylolisthesis. J Clin Neurosci 22(10):1619-1624
72. Cheng CL et al (1989) Anterior spinal fusion for spondylolysis and isthmic spondylolisthesis. Long term results in adults. J Bone Jt Surg Br 71(2):264-267

73. Riouallon $\mathrm{G}$ et al (2013) Outcomes of anterior lumbar interbody fusion in low-grade isthmic spondylolisthesis in adults: a continuous series of 65 cases with an average follow-up of 6.6 years. Orthop Traumatol Surg Res 99(2):155-161

74. Ishihara $\mathrm{H}$ et al (2001) Minimum 10-year follow-up study of anterior lumbar interbody fusion for isthmic spondylolisthesis. J Spinal Disord 14(2):91-99

75. Phan K et al (2015) Minimally invasive versus open transforaminal lumbar interbody fusion for treatment of degenerative lumbar disease: systematic review and meta-analysis. Eur Spine J 24(5):1017-1030

76. Carreon LY, Glassman SD, Howard J (2008) Fusion and nonsurgical treatment for symptomatic lumbar degenerative disease: a systematic review of Oswestry Disability Index and MOS Short Form-36 outcomes. Spine J 8(5):747-755

77. Glassman SD et al (2009) Lumbar fusion outcomes stratified by specific diagnostic indication. Spine J 9(1):13-21

78. Goldstein CL, Phillips FM, Rampersaud YR (2016) Comparative effectiveness and economic evaluations of open versus minimally invasive posterior or transforaminal lumbar interbody fusion: a systematic review. Spine (Phila Pa 1976) 41(Suppl 8):S74-S89

79. Kongsted A et al (2015) Patients with low back pain had distinct clinical course patterns that were typically neither complete recovery nor constant pain. A latent class analysis of longitudinal data. Spine J 15(5):885-894

80. Axén I, Leboeuf-Yde C (2013) Trajectories of low back pain. Best Pract Res Clin Rheumatol 27(5):601-612

81. Fung $\mathrm{CH}$ et al (2008) Systematic review: the evidence that publishing patient care performance data improves quality of care. Ann Intern Med 148(2):111-123

\section{Affiliations}

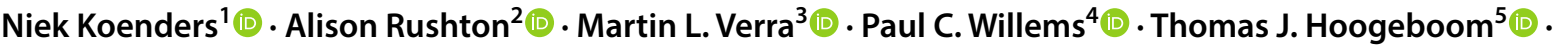 J. Bart Staal ${ }^{5,6}$ (i)}

Niek Koenders

niek.koenders@ radboudumc.nl

Alison Rushton

a.b.rushton@bham.ac.uk

Martin L. Verra

martin.verra@insel.ch

Paul C. Willems

p.willems@mumc.nl

Thomas J. Hoogeboom

thomas.hoogeboom@ radboudumc.nl

J. Bart Staal

bart.staal@radboudumc.nl

1 Orthopaedics Department, Radboud Institute for Health Sciences, Radboud University Medical Center, Nijmegen, The Netherlands
2 Centre of Precision Rehabilitation for Spinal Pain, School of Sport, Exercise and Rehabilitation Sciences, College of Life and Environmental Sciences, University of Birmingham, Birmingham, UK

3 Department of Physiotherapy, Insel Group, Bern University Hospital, Bern, Switzerland

4 Maastricht University Medical Centre, Maastricht, The Netherlands

5 IQ Healthcare, Radboud Institute for Health Sciences, Radboud University Medical Center, Nijmegen, The Netherlands

6 Research Group Musculoskeletal Rehabilitation, HAN University of Applied Sciences, Nijmegen, The Netherlands 